\title{
What the guide does not tell you: Reflections on and lessons learned from applying the COM-B behaviour model for designing real life interventions
}

\begin{abstract}
Background: Substantial inconsistency exists in the effectiveness of existing interventions to improve heart failure (HF) self-care, which can be partially explained by the fact that selfmanagement interventions often lack theoretical models that underpin intervention development. The COM-B behaviour model is a comprehensive theoretical framework that can be used to develop effective, theory-based interventions.

Purpose: The aim of this article is to highlight the challenges and practical solutions when applying the COM-B model to HF self-care, in order to provide useful support for researchers intending to use the model for designing behaviour change interventions.

Methods: 'The Behaviour Change Wheel' handbook provides a step-by-step guide to understand and change behaviour. When following the guide, some practical and methodological challenges were encountered. Lessons learnt to overcome these challenges are reported.

Results (Discussion): Although the handbook is a comprehensive guide for designing behaviour change interventions, a number of challenges arose. For example, the descriptions provided in the guide were not always sufficient to make solid judgments on how to categorise determinants; narrowing down intervention possibilities to a manageable number and prioritising potential behaviour change techniques over others involved a certain amount of subjectivity in an otherwise highly systematic and structured approach. For the encountered challenges, solutions are provided to illustrate how the model was applied practically to design theory-based behaviour change interventions.
\end{abstract}


Conclusions: This paper provides a useful reference for researchers' use of the COM-B behaviour model, as it outlines challenges that may occur and potential solutions to overcome them.

\section{Keywords}

COM-B behaviour model, behaviour change wheel, methodological discussion, complex intervention design, lessons learned 


\section{Introduction}

Numerous systematic reviews investigating the efficacy and effectiveness of specific selfmanagement interventions applying behaviour change techniques (BCTs) have been published [1-4]. The results of these studies, however, remain largely inconsistent. Part of this inconsistency can be explained by the fact that these interventions often lack underlying theoretical models [5]. A review of self-management interventions promoting self-care of heart failure (HF) patients concluded that only very few studies used explicit theory-based interventions; the majority lacked specification of the mechanisms employed to improve selfcare [5]. This echoes the critique articulated by Michie and colleagues [6], who stated that interventions are frequently designed without applying an underlying evidence-based model.

Many interventions are designed following the 'ISLAGIATT' principle, a term coined by Martin Eccles, Emeritus Professor of Clinical Effectiveness at Newcastle University. The acronym stands for 'It seemed like a good idea at the time'. It reflects a widespread approach to intervention design where the strategy is known from the outset, without having conducted a thorough investigation of the behaviour that needs to be targeted. Instead, intervention designers tend to use their personal experience, a cursory analysis or implicit common sense models for designing interventions [7]. Such efforts are likely to result in what Chalmers and Glasziou refer to as 'research waste', i.e. research in which insufficient effort has been made to fully understand the behaviour that needs to change before developing an intervention [8].

High quality evidence in the form of a systematic review of reviews of interventions associated with increased effectiveness highlights that behaviour change interventions (BCIs) are most 
effective if they use established, well-defined BCTs [9]. Although there have been several attempts to apply a more systematic approach to intervention design including MINDSPACE [10], none of the frameworks cover all relevant components needed to develop complex BCIs. The Capability, Opportunity and Motivation (COM-B) model of behaviour [7] is an integrative theoretical framework that provides a systematic method of identifying target behaviours and techniques judged to be most effective in changing behaviour.

Over the years, the COM-B model has gained momentum among intervention designers applying it to various health-related contexts. 'The Behaviour Change Wheel: A Guide to Designing Interventions' (BCW) handbook (hereafter referred to as "the guide") is a comprehensive stepby-step guide to designing interventions [7]. The article published in TBM by Webster and colleagues on the application of the COM-B model to improving condom use provides a practical example of the exceptional usefulness of this model, with which we fully agree [11]. Still, we encountered some practical and methodological challenges when applying it. To date, a plethora of articles exist that describe the application of the COM-B model as a framework for designing BCIs [12-19]. Out of these articles, however, only very few refer briefly to the challenges associated with its use [12,13].

The essay by Tate and colleagues considered the potential advantages and challenges of applying the COM-B model [20]. We would like to build on this by reporting in more depth the challenges that may arise during practical application of the model, along with how these challenges can be overcome. To our knowledge, this is the first practical example that focuses solely on the challenges of applying the COM-B model to designing a 'real life' intervention, that is, an 
intervention that is intended to be implemented in a real-world setting [21]. The aim of this commentary is to address these challenges and outline potential solutions, to further improve the effective use of the COM-B model in future research, and with that the successful impact of behavior change interventions.

\section{Discussion}

This article is a result of the authors' reflections on the behaviour change intervention design process. Intervention development followed 'The Behaviour Change Wheel' guide mentioned above. The discussion draws on experiences from a research project [22], which applied the eight steps described in the guide as the underlying framework for designing BCIs for HF self-care. Barriers to HF self-care were extracted from two comprehensive meta-studies, one qualitative meta-summary [23] and one quantitative meta-analysis [24]. The challenges encountered, along with the chosen solutions, provide the content of the following discussion.

The challenges outlined below were encountered regarding step 4 "Identify what needs to change" and step 7 "Identify behaviour change techniques". First, the title and key aspects of what each step entails are described (in italics). We then discuss the methodological steps by juxtaposing the challenges we encountered and our deliberations involved. Finally, we offer practical solutions for how we resolved the issues to assist future intervention designers in facilitating the process. In the conclusion, we summarise our key experiences with employing the COM-B model.

\section{Identify what needs to change}


This step deals with identifying what needs to change according to the COM-B model to achieve the desired behaviour change. The COM-B model components 'Capability', 'Opportunity' and 'Motivation' all influence and in turn are influenced by 'Behaviour'. Each target behaviour must be classified according to the COM-B components to identify the domain that should be targeted for a behaviour to change in the desired direction [7].

This step was completed by mapping the barriers to HF self-care onto the six COM-B components. Challenges were encountered in relation to categorising the determinants.

Challenge 1: The descriptions and examples provided in the guide were not always sufficient to make solid judgements on how to categorise determinants according to the COM-B model. We encountered situations in which two COM-B components seemed equally applicable for one determinant. For example, the behavioural determinant 'self-efficacy' was extracted as a barrier to HF self-care from the qualitative meta-summary [23]. The sentence reads:

"Some patients had low self-efficacy, considered the clinic responsible for drug management and expected to receive help from health professionals."

Since no further contextual information is available in the original article, self-efficacy could be classified as 'psychological capability'. In the guide, it is defined as "knowledge or psychological skills, strength or stamina to engage in the necessary mental processes" [7, p.63]. Self-efficacy could also, however, be classified as a construct related to 'reflective motivation' because it involves evaluation about oneself. According to the guide, 'reflective motivation' is defined as 
"reflective processes involving plans (self-conscious intentions) and evaluations (beliefs about what is good and bad)" [7, p.63].

Solution 1: In case of disagreement, rationale and possibilities were discussed among research team members until a solution was found. We discussed 'reflective motivation' as the best categorisation choice for self-efficacy since it requires knowledge and skills, but also the ability to reflect on and evaluate whether one feels these skills are sufficient to perform the necessary behaviour. Our discussion led to the realisation that self-efficacy is not only about having knowledge or skills, but involves a self-reflective process, regardless of whether or not this process is fully conscious. The lack of knowledge or skills to engage in the needed behaviour without this self-reflection would be categorised as 'psychological capability'. It might be reflected as uncertainty about performing the behaviour rather than self-efficacy. An example of uncertainty was seen in the following barrier to self-care:

"...uncertainty, misconceptions and lack of patients' understanding were common factors hampering the execution of self-care activities, partly because patients did not know what to ask or did not dare to ask."

In accordance with the recommendation by Sinnott and colleagues [13], we recorded the options available and provided explanations for our decisions in order to improve the transparency of the subjective decisions involved in categorising the determinants. 
=> Lesson learned: As a suggestion for improving the guide, it would be helpful to discern uncertainty (psychological capability) from self-efficacy (reflective motivation), since the two are closely related, but ultimately distinct.

Challenge 2: The COM-B model does not take into account cultural background, age or other factors that might be relevant for designing BCIs; hence users of the COM-B model need to fill in this missing information. For instance, the model does not help to discern whether cultural background is influencing a specific behaviour. This is a judgment that rests solely with the researchers. We illustrate this point by the following example taken from the qualitative metasummary [23]:

"Beliefs act as a barrier to self-care if patients do not attribute their symptoms to HF, have wrong assumptions about what causes HF or which factors or self-care actions influence symptoms..."

Both researchers agreed that a patient's attribution or assumptions of what causes or influences HF symptoms were related to 'psychological capability'. According to the guide, 'psychological capability' is defined as "having the knowledge, psychological skills, strength or stamina to perform the behaviour" [7, p.59]. When it came to 'beliefs', however, we faced disagreement. One researcher thought that 'beliefs' should be categorised as 'social opportunity' because beliefs are often shaped or informed by cultural norms. 'Social opportunity' is defined as "opportunity afforded by interpersonal influences, social cues and cultural norms that influence the way we think about things" [7, p.63]. However, the other researcher argued that 'beliefs' should be 
categorised as 'reflective motivation' because one's beliefs are determined by a reflective evaluation of what is right. According to the guide, 'reflective motivation' is defined as "reflective processes involving plans (self-conscious intentions) and evaluations (beliefs about what is good and bad)" [7, p.63].

Solution 2: We concluded that 'beliefs' in this context should be categorised as 'reflective motivation', because even if culturally influenced, individual evaluation is required to shape the way one attributes illness symptoms. We further concluded that 'social opportunity', which encompasses cultural norms, should be treated as a potential underlying contextual factor, even if they are not explicitly stated. For example, we considered 'social opportunity' as a relevant contextual factor, which may be shaping the evaluation process of symptoms, even when it is not necessarily obvious from the explicit information.

=>Lesson learned: For further clarity, it might be useful to include more terms on the list of relevant behaviours for each of the COM-B components in the guide. Currently, 'reflective motivation' entails reflective processes such as planning (self-conscious intentions) and evaluations (beliefs about what is good and bad), while 'automatic motivation' includes emotional reactions, desires, impulses, inhibitions, drive states and reflex responses. Based on our knowledge, we suggest that under 'reflective motivation', perceptions, judgments, selfefficacy and expectations could be included, while under 'automatic motivation', personality traits, desires and inertia could be added. We acknowledge that whatever approach has been agreed, it needs to be consistently applied throughout the application of the model. 


\section{Identify behaviour change techniques (BCTs)}

This step identifies relevant BCTs for delivering the selected intervention functions. A BCT is an active component of an intervention designed to change behaviour. There are 93 BCTs listed on the BCT Taxonomy (v1). It is important to first identify all possible BCTs and then narrow down the 'long list' to select the BCTs. The guide offers two suggestions: (1) selecting the BCTs used most frequently in the literature, (2) using the APEASE criteria. The APEASE criteria helps determine whether a BCT is affordable, practicable, effective/cost-effective, acceptable, shows side-effects/safety and equitable [7].

All potentially relevant BCTs were selected based on the list provided in the guide. The list shows which BCTs are appropriate for each intervention function. We then narrowed down the 'long list' by logical self-imposed rules, followed by application of the APEASE criteria. This step posed the following challenges.

Challenge 3: How to best narrow down the 'long list' of potential BCTs to a manageable number that could be used for intervention creation.

Solution 3: We systematically applied the following self-imposed rules to narrow down the list of potential BCTs. First, we gave preference to the most frequently used BCTs according to the guide (highlighted in the guide in bold) [7]. Second, overlapping determinants with the same BCTs were combined. Third, HF patients' preferences were sought when multiple BCTs were available for one determinant (barrier to self-care) to help decide which of the BCTs could be 
successfully integrated into the every-day life of patients. Therefore, $33 \mathrm{HF}$ patients were asked to rate different BCTs in relation to its likeliness of use applying a 4-point Likert scale ranging from 0 ('Not likely at all”) to 3 ('Extremely likely"). Finally, we created 'intervention bundles' by combining two or more BCTs that fit together in a complementary logical sequence. For example, to overcome the barrier of attributing HF symptoms to existing comorbidities or medication side-effects, the BCT 'Information about health consequences' was combined with 'Behavioural practice/rehearsal' to form one intervention bundle.

Challenge 4: Although Ojo and colleagues [25] highlight the importance of applying the APEASE criteria to determine the most appropriate intervention function, we experienced difficulty when applying it to further reduce the list of potential BCTs. In particular, it was difficult to judge accurately whether changing a specified behaviour will be considered acceptable by end users or what degree of effectiveness it will have.

Solution 4: Since we were unable to make qualified judgements regarding the APEASE criteria ourselves, we engaged in additional interviews with stakeholders to help us better judge the appropriateness of the potential BCTs [21].

\section{Conclusions}

We found that the handbook by Michie and colleagues [7] is a practical, accessible and wellstructured guide for designing BCIs. Since it is impossible for any guide to cover all potential situations that researchers may encounter, this article aimed to provide further support to future users of the COM-B model. Despite the highly systematic and structured approach of the COM- 
B model, there were challenges associated with its use. Throughout the process, we had to make subjective and pragmatic decisions to fill in the missing information, which seems at odds with an otherwise entirely scientific approach. We agree with the critique by that the use of the framework is time consuming to apply, particularly where there are multiple target behaviours [11]. However, we are of the opinion that it is time well invested, because it results in explicit BCTs which allows intervention replication, and the systematic approach heightens the chances of intervention success in both individual and broader policy settings [26].

Furthermore, some of the BCTs listed in the BCT taxonomy (v1) could be intimidating to patients and even alienate the relationship between patient and healthcare provider. For example, BCTs such as 'anticipated regret', 'vicarious consequences' or 'salience of consequences' frequently work on the grounds of punishment, deterrence or guilt-tripping. From a clinical perspective, we suggest moving from a world of sticks to a world of carrots by changing the perspective from looking at patients' deficits or shortcomings to focusing on patients' resources or strengths. For instance, rather than providing information about negative health consequences when omitting a recommended self-care behaviour (e.g. not taking diuretics can lead to fluid retention), we should rather encourage patients by providing information about positive health consequences when self-care is executed (e.g. taking diuretics can help relieve swelling in the legs and ankles). This positive focus is also supported by the scientific literature. Although deterrence threads are perceived as essential mechanisms for influencing behaviour, they are often ineffective. More recent findings indicate that susceptibility to deterrence is largely dependent upon key individual differences, including morality and self-control [27]. 
We hope that sharing our reflections on and lessons learned from applying the COM-B model supports intervention designers and encourages neophytes in the field of intervention design to enter this stimulating field with more confidence and understanding, and to continue their endeavour despite challenging situations. 


\section{References}

1. Jovicic, A., Holroyd-Leduc, J. M., \& Straus, S. E. (2006). Effects of self-management intervention on health outcomes of patients with heart failure: a systematic review of randomized controlled trials. BMC cardiovascular disorders, 6(1), 43.

2. Ditewig, J. B., Blok, H., Havers, J., \& van Veenendaal, H. (2010). Effectiveness of selfmanagement interventions on mortality, hospital readmissions, chronic heart failure hospitalization rate and quality of life in patients with chronic heart failure: a systematic review. Patient education and counseling, 78(3), 297-315.

3. Pfaeffli Dale, L., Dobson, R., Whittaker, R., \& Maddison, R. (2016). The effectiveness of mobile-health behaviour change interventions for cardiovascular disease self-management: a systematic review. European journal of preventive cardiology, 23(8), 801-817.

4. O’Dwyer, T., Maguire, S., Mockler, D., Durcan, L., \& Wilson, F. (2019). Behaviour change interventions targeting physical activity in adults with fibromyalgia: a systematic review. Rheumatology international, 39(5), 805-817.

5. Barnason, S., Zimmerman, L., \& Young, L. (2012). An integrative review of interventions promoting self-care of patients with heart failure. Journal of Clinical Nursing, 21(3-4), 448475.

6. Michie, S., van Stralen, M.M., \& West, R. (2011). The behaviour change wheel: a new method for characterising and designing behaviour change interventions. Implement Science, 6:42.

7. Michie, S., Atkins, L., \& West, R. (2014). The behaviour change wheel: a guide to designing interventions. UK: Silverback Publishing. 
8. Chalmers, I., \& Glasziou, P. (2009). Avoidable waste in the production and reporting of research evidence. Lancet, 374(9683), 86-89.

9. Greaves, C.J., Sheppard, K.E., Abraham, C., Hardeman, W., Roden, M., Evans, P.H., \& Schwarz, P. (2011). Systematic review of reviews of intervention components associated with increased effectiveness in dietary and physical activity interventions. BMC Public Health, 11:119.

10. Institute for Government. (2010). MINDSPACE: Influencing behaviour through public policy. Institute for Government, the Cabinet Office. Retrieved from https://www.instituteforgovernment.org.uk/sites/default/files/publications/MINDSPAC E.pdf

11. Webster, R., Michie, S., Estcourt, C., Gerressu, M. \& Bailey, J.V., \& MenSS Trial Group. (2015). Increasing condom use in heterosexual men: development of a theory-based interactive digital intervention. Translational Behavioral Medicine, 6(3), 418- 427.

12. Flannery, C., McHugh, S., Anaba, A.E., Clifford, E., O'Riordan, M., Kenny, L.C., McAuliffe, F.M., Kearney, P.M., \& Byrne, M. (2018). Enablers and barriers to physical activity in overweight and obese pregnant women: an analysis informed by the theoretical domains framework and COM-B model. BMC Pregnancy and Childbirth, 18(1):178.

13. Sinnott, C., Mercer, S.W., Payne, R.A., Duerden, M., Bradley, C.P., \& Byrne, M. (2015). Improving medication management in multimorbidity: development of the MultimorbiditY COllaborative Medication Review And DEcision Making (MY COMRADE) intervention using the Behaviour Change Wheel. Implement Science, 10:132. 
14. Cowdell, F., \& Dyson, J. (2019). How is the theoretical domains framework applied to developing health behaviour interventions? A systematic search and narrative synthesis. BMC public health, 19(1), 1180.

15. Ellis, K., Pears, S., \& Sutton, S. (2019). Behavioural analysis of postnatal physical activity in the UK according to the COM-B model: a multi-methods study. BMJ open, 9(8), e028682.

16. Grant, A., Morgan, M., Mannay, D., \& Gallagher, D. (2019). Understanding health behaviour in pregnancy and infant feeding intentions in low-income women from the UK through qualitative visual methods and application to the COM-B (Capability, Opportunity, Motivation-Behaviour) model. BMC pregnancy and childbirth, 19(1), 56.

17. Jennings, H. M., Morrison, J., Akter, K., Kuddus, A., Ahmed, N., Kumer Shaha, S., Nahar T., Haghparast-Bidgoli, H., Azad Kahn, AK., \& Fottrell, E. (2019). Developing a theorydriven contextually relevant mHealth intervention. Global health action, 12(1), 1550736

18. McDonagh, L. K., Saunders, J. M., Cassell, J., Curtis, T., Bastaki, H., Hartney, T., \& Rait, G. (2018). Application of the COM-B model to barriers and facilitators to chlamydia testing in general practice for young people and primary care practitioners: a systematic review. Implementation Science, 13(1), 130.

19. Smith, C. A., McNeill, A., Kock, L., \& Shahab, L. (2019). Exploring mental health professionals' practice in relation to smoke-free policy within a mental health trust: a qualitative study using the COM-B model of behaviour. BMC psychiatry, 19(1), 54.

20. Tate, D. F., Lytle, L. A., Sherwood, N. E., Haire-Joshu, D., Matheson, D., Moore, S. M., Loria, C.M., Pratt, C., Ward, D.S., Belle, S.H., \& Michie, S. (2016). Deconstructing interventions: approaches to studying behavior change techniques across obesity interventions. Translational behavioral medicine, 6(2), 236-243. 
21. XXXX, YYYY (reference removed for peer reviewing process to remain anonymous)

22. XXXX, YYYY (reference removed for peer reviewing process to remain anonymous)

23. Herber, O.R., Bücker, B., Metzendorf, M.-I., \& Barroso, J. (2017). A qualitative metasummary using Sandelowski and Barroso's method for synthesizing qualitative research to explore barriers and facilitators to self-care in heart failure patients. European Journal of Cardiovascular Nursing, 16(8), 662-677.

24. Kessing, D., Denollet, J., Widdershoven, J., \& Kupper, N. (2016). Psychological determinants of heart failure self-care: systematic review and meta-analysis. Psychosomatic Medicine, 78(4), 412-431.

25. Ojo, S.O., Bailey, D.P., Brierley, M.L., Hewson, D.J., \& Chater, A.M. (2019). Breaking barriers: using the behavior change wheel to develop a tailored intervention to overcome workplace inhibitors to breaking up sitting time. BMC Public Health, 19(1):1126.

26. West, R., Michie, S., Atkins, L., Chadwick, P., \& Lorencatto, F. (2019). Achieving behavior change: A guide for local government and partners. Public Health England Publications.

27. Fine, A., \& van Rooij, B. (2017). For whom does deterrence affect behavior? Identifying key individual differences. Law and Human Behavior, 41(4), 354-360. 